\title{
VIABILIDAD Y GERMINACIÓN DE LAS SEMILLAS DE ABIES PINSAPO BOISS.
}

\author{
Montserrat ARISTA, Salvador TALAVERA y Javier HERRERA
}

\begin{abstract}
RESUMEN: Se estudió la viabilidad y germinación de las semillas de Abies pinsapo Boiss. sobre una muestra de 2000 piñones procedentes de dos árboles de la Serranía de Grazalema. Las semillas son muy variables en tamaño, tanto dentro de un mismo indivíduo como entre plantas diferentes. Además, un alto porcentaje de piñones carecen de embrión y son por tanto inviables (normalmente los menores de $60 \mathrm{mg}$ ). Las semillas mayores de $60 \mathrm{mg}$ alcanzaron un $33 \%$ de germinación, mientras que las menores de ese tamaño sólo llegaron al $8 \%$. En conjunto, sólo germinó el $26 \%$ de todas las semillas $(\mathrm{N}=1280)$. Dañar las bolsas de resina que posee el piñón redujo el porcentaje al $0.2 \%$. Los piñones germinan relativamente pronto cuando se dan las condiciones necesarias. En caso de no germinar el primer año, parecen no poder hacerlo en años sucesivos.
\end{abstract}

Palabras clave: Abies pinsapo, Viabilidad, Germinación, Grazalema, Andalucía.

ABSTRACT: Size, viability and germination were studied in a sample of 2000 seeds from two individuals of the relict endemic Abies pinsapo Boiss. growing in Serranía de Grazalema (southern Spain). Seed mass and viability showed considerable variation both within and among trees. A large proportion of unfilled, mostly empty seeds lighter than $60 \mathrm{mg}$ was found with a germination rate as low as $8 \%$. Seeds heavier than $60 \mathrm{mg}$ germinated at much higher rate $(33 \%)$. As a whole, $26 \%$ of all seeds $(\mathrm{N}=1280)$ germinated.Seeds with manually injured testas loose virtually all germination capacity $(0.2 \%)$. Seeds germinate soon after dispersal if the necessary conditions are met, while germination seems unlikely on subsequent years.

Key words: Abies pinsapo, Seed-viability, Germination, Grazalema, Andalucía.

\section{INTRODUCCIÓN}

Abies pinsapo Boiss. es un abeto endémico de las montañas andaluzas cuyas mayores poblaciones se encuentran en las serranías de Grazalema (Cádiz) y Ronda (Málaga). Aunque se trata de una especie rara y considerablemente protegida, lo cierto es que hay pocos datos sobre su biología. Así, aunque hay información sobre su fitoquímica (Barrero et al., 1989), se ignoran la mayoría de las particularidades de su reproducción. Ello contrasta con los abundantísimos datos que hay referidos a otras especies de Abies (por ejemplo, Barbour et al., 1990; Bert y Becker, 1990; Fady 1990; Neale y Adams, 1985; Pauley y Clebsch, 1990; Schroeder, 1989; Shea, 1989; Szwagrzyk, 1990; Zoladeski y Maycock, 1990). 
En este trabajo se presentan datos sobre germinación de semillas de A.pinsapo en el laboratorio como parte de un estudio a largo plazo sobre la ecología reproductiva y dinámica poblacional de esta especie (Arista, inédito). Se examinan los porcentajes de viabilidad y sus variaciones entre indivíduos, así como el efecto del sustrato sobre la germinación. También se examina el posible efecto negativo sobre la germinación de dañar mecánicamente la testa (Martin Bolaãos, 1947).

\section{MATERIAL Y MÉTODOS}

En el estudio se emplearon dos lotes de semillas recolectadas en el Parque Natural de la Sierra de Grazalema (Cádiz). Los lotes procedían de sendos indivíduos adultos situados dentro de la Reserva del Pinsapar. El indivíduo que en los sucesivo llamaremos Arbol 1 se encuentra en las laderas de la Sierra de Zafalgar, aproximadamente a $850 \mathrm{msm}$. El que llamaremos Arbol 2 se sitúa en la zona de Las Majadillas, a $750 \mathrm{msm}$. La distancia entre estos árboles es de $1.4 \mathrm{~km}$. Las piñas se recolectaron cuando empezaban a disgregarse en Octubre de 1989 y se llevaron al laboratorio, donde se separaron todos los piñones. Se consiguió así una muestra de aproximadamente 2200 semillas, 1100 por cada árbol. Los piñones se almacenaron en el frigorífico hasta Febrero de 1990, en que comenzaron los experimentos.

Para tener una idea de la variación de tamaño de las semillas, tanto dentro de cada planta como entre plantas, se tomaron al azar 100 piñones de cada árbol y se pesaron individualmente en una balanza electrónica con precisión de $1 \mathrm{mg}$. Tras anotar el peso cada piñón se abría y diseccionaba para comprobar si tenía embrión o no. Los piñones restantes (unos 2000) se pesaron individualmente y se agruparon por clases de tamaño.

La germinación se comprobó, por clases de tamaño, en cuatro situaciones posibles resultantes de combinar dos tipos de tratamiento: sustrato (tierra o papel) y daño mecánico de la testa (pinchando o no con una aguja las bolsas de resina). En cada tratamiento se incluyó un número parecido de semillas, hasta dar un total de 1280 . Los piñones se colocaron dentro de placas Petri (10 por placa) provistas de dos capas de papel de filtro, o bien rellenas de tierra procedente del Pinsapar. En ambos casos las placas se mantenían tapadas y los sustratos se conservaban constantemente húmedos con riegos periódicos. Todas las placas se colocaron en un laboratorio con luz artificial durante $10 \mathrm{~h}$ al día, y a una temperatura que no bajó de $18^{\circ} \mathrm{C}$. Se hicieron en total dos siembras en las mismas condiciones, una en los primeros días de Marzo y otra a comienzos de Abril de 1990. Semanalmente las placas se abrían para retirar y contar los piñones germinados (raíz mayor de $1 \mathrm{~mm}$ ). Los piñones que no germinaron en 1990 se guardaron otra vez en el frigorífico hasta Febrero de 1991 en que volvieron a sembrarse en placas Petri. Las condiciones de luz y temperatura fueron las mismas, pero sólo se empleó como sustrato papel de filtro.

\section{RESULTADOS}

\section{Tamaño y viabilidad de las semillas.}

La distribución por clases de tamaño de los piñones aparece en la Figura 1. Se observa una clara discordancia entre la distribución para uno y otro árbol, con un predominio de las semillas relativamente grandes en el Arbol 1, y al contrario en el Arbol 2. La diferencia entre ambas distribuciones es altamente significativa $\left(\chi^{2}=53.21\right.$, $\mathrm{p}<<0.001)$. 


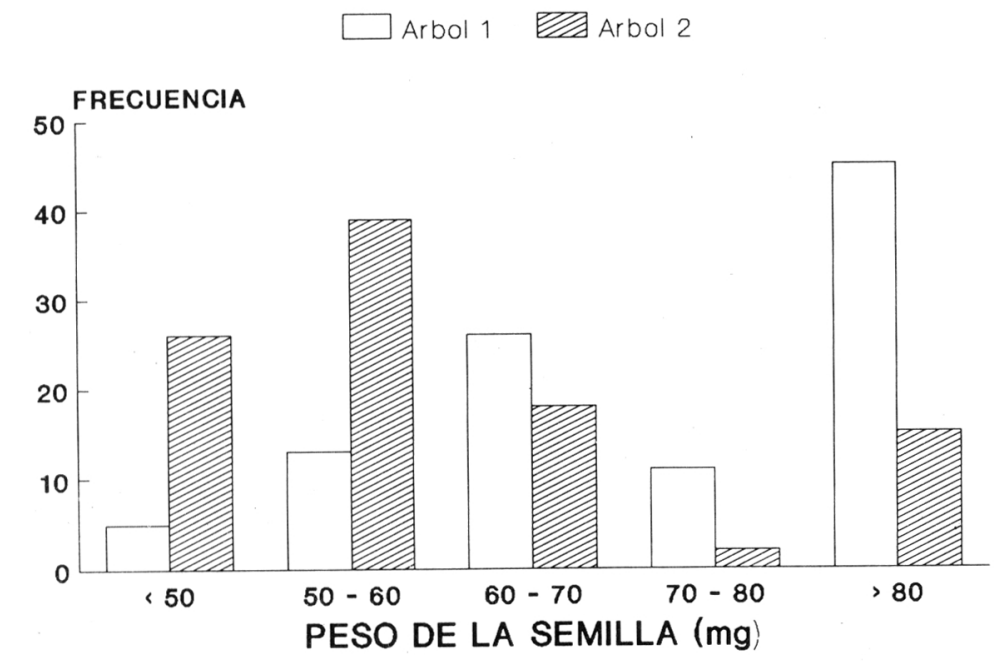

Figura 1. Distribución por clases de tamaño de piñones de Abies pinsapo procedentes de dos árboles del Pinsapar (Cádiz).

Al diseccionar un total de 200 semillas (100 por árbol) se observó que un alto porcentaje de ellas era totalmente inviable por carecer de embrión (Tabla 1). Para el Arbol 1 la proporción de semilla inviable fue del $41 \%$, y para el Arbol 2 del $63 \%$. Además se comprobó que la presencia o ausencia de embrión puede ser hasta cierto punto predicha a partir del peso del piñón, de manera que las semillas menores de 60 mg nunca lo poseían, mientras que las mayores de 60 con frecuencia (sobre todo en el Arbol 1) sí tenían embrión. Además, de un total de 230 piñones procedentes de ambos árboles que fueron sembrados sobre papel de filtro húmedo tan sólo germinaron $20(8.7 \%)$, lo que todavía refuerza más la idea de que esas semillas menores de $60 \mathrm{mg}$ son básicamente inviables. En vista de esto, en los experimentos realizados posteriormente sobre germinación sólo se han tenido en cuenta las semillas mayores de $60 \mathrm{mg}$.

\begin{tabular}{cccc}
\hline & & \multicolumn{2}{c}{ PESO DE LA SEMILLA $(\mathrm{mg})$} \\
\hline \multirow{2}{*}{ Árbol } & Embrión & $<60$ & $>60$ \\
\hline 1 & + & 0 & 59 \\
& - & 18 & 23 \\
\hline & + & 0 & 17 \\
& - & 65 & 18
\end{tabular}

Tabla 1. Número de semillas con o sin embrión para dos indivíduos de Abies pinsapo. $\mathrm{N}=100$ para cada uno de los árboles. 


\section{Germinación.}

En la Tabla 2 se expone la proporción de semillas mayores de $60 \mathrm{mg}$ que germinaron en varias combinaciones de dos tipos de tratamiento (sustrato y acción mecánica sobre la testa). En esta tabla se han juntado las semillas de los árboles 1 y 2 ya que, una vez que se descartan los piñones inviables, no hay diferencias significativas en la tasa de germinación de uno y otro indivíduo $\left(\mathrm{x}^{2}=1.19, \mathrm{p}=0.27\right)$.

\begin{tabular}{lccc}
\hline & & \multicolumn{2}{c}{ GERMINACION } \\
\hline Sustrato & Pinchados & + & - \\
\multirow{2}{*}{ Tierra } & + & 0 & 230 \\
& - & 140 & 160 \\
Papel & + & 1 & 249 \\
& - & 173 & 97 \\
\hline
\end{tabular}

Tabla 2. Germinación de piñones de Abies pinsapo en dos tipos de sustrato según que se hayan dañado o no las bolsas de resina que poseen en la testa.

La acción mecánica sobre las bolsas de resina que poseen las semillas redujo drásticamente su capacidad de germinación. De un total de 480 piñones a los que se aplicó dicho tratamiento tan sólo uno $(0.2 \%)$ germinó (Tabla 2).

El porcentaje de germinación de las semillas intactas sobre papel fue del $33 \%$, y tan sólo del $26 \%$ en tierra (Tabla 2). Aunque débil, existe un efecto significativo del sustrato (tierra o papel) sobre la tasa de germinación de los piñones intactos $\left(\chi^{2}=6.22\right.$, $\mathrm{p}=0.01)$.



Figura 2. Germinación de dos tandas de semillas de Abiés pinsapo sembradas en placas de Petri con papel de filtro húmedo ('Papel') y en placas con tierra procedente del Pinsapar ('Tierra'). Las flechas señalan el momento de la siembra de la primera y segunda tanda de piñones. 
En la Figura 2 se ha representado el número acumulado de semillas germinadas semanalmente para dos tandas de piñones intactos procedentes de los árboles 1 y 2. La siembra de la primera tanda precedió a la de la segunda aproximadamente en un mes. Se observa que, como se dijo antes, los piñones sembrados sobre papel germinan en mayor cantidad. En el caso de la primera tanda, además, la germinación sobre papel es significativamente más rápida que sobre tierra (test de Kolmogorov-Smirnov, $\mathrm{D}=$ $0.315, \mathrm{p}<0.001)$. En la segunda tanda no se produce este fenómeno de forma estadísticamente significativa $(\mathrm{D}=0.036, \mathrm{p}>0.5)$.

Cuarenta piñones intactos mayores de $60 \mathrm{mg}$ que no germinaron en 1990 volvieron a sembrarse sobre papel en Febrero de 1991. Un més después ninguno de ellos había germinado. Por otro lado, de 422 piñones cuyas bolsas de resina habían sido pinchadas en 1990 y no germinaron en dicho año, tampoco ninguno lo hizo en 1991 .

\section{DISCUSIÓN}

Los dos ejemplares de Abies pinsapo en los que se ha centrado este estudio mostraban una baja proporción de semilla viable en la cosecha del año 1989 (Figura 1). Esto no es excepcional si se compara con los datos aportados anteriormente para otras especies del género Abies. Barton (1930) dio valores del $69 \%$ en A. arizonica, y Schröeder (1989) del $24 \%$ al $92 \%$ en A. alba. En otras especies de gimnospermas también es un hecho común la producción de numerosas semillas sin embrión, lo que suele estar relacionado con fenómenos de polinización insuficiente, autogamia, etc. (Allison, 1990; Smith et al., 1988). Los estudios realizados hasta la fecha por nosotros en el Pinsapar indican que en el caso de $A$. pinsapo también tienen lugar estos fenómenos (Arista, inédito). Ello puede tener implicaciones a la hora de realizar siembras dirigidas a la repoblación, ya que la utilización de semillas no seleccionadas tendrá seguramente como consecuencia una baja germinación.

La gran diferencia de viabilidad entre las semillas del Arbol 1 y las del Arbol 2 señaladas en el presente estudio indican, además, que son de esperar variaciones interindividuales muy grandes (ver también Schröeder, 1989). Ello sugiere que también las plantas madre deberían seleccionarse cuidadosamente para que las siembras tengan el mayor éxito posible. Los árboles en los que se observe una mayor proporción de piñones mayores de $60 \mathrm{mg}$ serán sin duda los más adecuados para emplearse como fuentes de semilla. Naturalmente, la utilización del valor de $60 \mathrm{mg}$ como predictor de la viabilidad se debe a que, en promedio, ese es el peso de la testa más el ala, de manera que por encima de ese valor la semilla contendrá probablemente embrión y por debajo no lo tendrá.

La rotura de las bolsas de resina que presenta el piñón tiene un efecto absolutamente negativo sobre la germinación (Tabla 2). El por qué de este hecho no se puede dilucidar con los resultados del presente estudio, aunque es posible que se deba a la liberación de sustancias inhibidoras. Sí podemos afirmar que la ausencia de germinación no se debe a una mala penetración de agua en la semilla, ya que hemos observado que los piñones tratados se hinchan exactamente igual que los intactos. En cualquier caso, y sea cual sea el mecanismo inhibidor, lo cierto es que desde el punto de vista práctico la manipulación de los piñones debe ser cuidadosa para evitar dañar la testa. De otra manera se pierde prácticamente toda posibilidad de éxito en la siembra. Este hecho puede también tener alguna implicación en el proceso de regeneración natural, si las semillas son alguna vez dispersadas por animales vertebrados capaces de dañar las bolsas de resina (Martín Bolaños, 1947).

El sustrato sobre el que se realizaron las siembras (papel de filtro o tierra 
húmedos) resultó tener una importancia relativamente pequeña en las tasas de germinación (Tabla 2, Figura 2). Esto sugiere que la tierra del Pinsapar no produce efectos alelopáticos, o bien que éstos son pequeños en las condiciones del estudio. De hecho, la menor germinación sobre tierra debe achacarse a que las semillas son más atacadas por hongos del suelo y se pudren antes de germinar, mientras que el medio estéril que representa el papel elimina esta fuente de mortalidad. Señalemos finalmente que los piñones de Abies pinsapo germinan tan pronto como se dan las condiciones para ello, o no lo hacen ya en años sucesivos. Esto descarta la existencia de fenómenos de dormancia y elimina la posibilidad de que en la naturaleza se formen bancos permanentes de semillas enterradas. La aparición de las plántulas en el campo se producirá en oleada poco después de la dispersión de los piñones. Las observaciones realizadas hasta ahora confirman esta idea (Arista, inédito).

AGRADECIMIENTOS. La Agencia de Medio Ambiente (Junta de Andalucía) facilitó permiso para trabajar en el Pinsapar (Parque Natural de Grazalema) y proporcionó parte (Arbol 2) de los piñones empleados en este estudio.

\section{BIBLIOGRAFÍA}

ALLISON, T.D. - 1990-Pollen production and plant density affect pollination and seed production in Taxus canadensis. Ecology, 71: 516-522.

BARBOUR, M.G., B.M. PAVLIK, \& J.A. ANTOS -1990- Seedling growth and survival of red and white fir in a Sierra Nevada ecotone. Amer. J. Bot. 77: 927-938.

BARRERO, A.F., J.F. SANCHEZ, E.J. ALVAREZ-MANZANEDA, \& M.M. DORADO -1989Sesquiterpenoids related to juvabione in Abies pinsapo. Phytochemistry, 28: 2617-2620.

BARTON, L.V. -1930- Hastening the germination of some coniferous seeds. Amer. J. Bot., 17: 88-115.

BERT, G.D. \& H.M. BECKER - 1990- Present and past vitality of silver fir (Abies alba Mill.) in the Jura Mountains - A dendroecological study. Ann. Sci. Forest., 47: 395-412.

FADY, B. -1990- Genetic variability of height growth components of the Greek Fir (Abies cephalonica). Can. J. Forest. Res., 20: 1453-1460.

MARTIN BOLAÑOS, M. -1947- Ensayo de investigación indirecta sobre origen, desarrollo y producciones del monte alto. Boletín del Instituto Forestal de Investigaciones y Experiencias, 34.

NEALE, D.B. \& W.T. ADAMS - 1985- Allozyme and mating-system variation in Balsam Fir (Abies balsamea) across a continuous elevational transect. Can. J. Bot., 63: 2448-2453.

PAULEY, E.F. \& E.E.C. CLEBSCH -1990- Patterns of Abies fraseri regeneration in a Great Smoky Mountains spruce-fur forest. Bull. Torrey Bot. Club, 117: 375-381.

SCHROEDER, S. - 1989-Oucrossing rates and seed characteristics in damaged natural populations of Abies alba Mill. Silvae Genetica, 38: 185-189.

SHEA, P.J. -1989- Interactions among phytophagous insect species colonizing cones of White Fir (Abies concolor). Oecologia, 81: 104-110.

SMITH, C.C., J.L. HAMRICK \& C.L. KRAMER - 1988- The effects of stand density on frequency of filled seeds and fecundity in lodgepole pine (Pinus contorta Dougl.). Canadian J. For. Res., 18: $453-460$.

SZWAGRZYK, J. -1990- Natural regeneration of forest related to the spatial structure of trees: a study of two forest communities in western Carpathians, southern Poland. Vegetatio, 89: 1122.

ZOLADESKI, C.A. \& P.F. MAYCOCK -1990- Dynamics of the boreal forest in northwestern Ontario. Amer. Midl. Naturalist, 124: 289-300.

(Aceptado para su publicación en octubre de 1991)

Dirección de los autores: Departamento de Biología Vegetal y Ecología. Universidad de Sevilla. Apartado 1095. 41080 Sevilla. 